\title{
Analyse expérimentale de la stabilité hydraulique de la turbine Francis à charge partielle
}

\author{
Experimental analysis of the hydraulic stability \\ of the partial load Francis turbine
}

\author{
P. Henry, M. Wegner et J. E. Graeser
}

\section{Introduction}

Les turbines Francis cumulent deux handicaps en ce qui concerne la stabilité de leur comportement.

Le premier réside dans la fixité angulaire des aubes du rotor qui entraîne des vorticités variables à l'entrée de l'aspirateur dès que l'on s'éloigne du point de fonctionnement optimum, le deuxième est créé par le coude de l'aspirateur qui engendre d'importants effets non axisymétriques dans l'écoulement aval.

Il en résulte de nombreux problèmes à l'étude desquels une abondante littérature est consacrée (voir bibliographie). Une classification schématique permet de répartir les contributions dans trois domaines.

Le premier concerne principalement la description expérimentale des écoulements périodiques intervenant dans les modèles réduits et les prototypes de turbines Francis, ainsi que leurs répercussions sur le comportement de la machine, $[1,2,3,4,5]$.

Le deuxième domaine s'intéresse plus précisément aux paramètres gouvernant les causes des écoulements périodiques, stables ou non (formes géométriques, conduits hydrauliques, degré de cavitation). Il englobe la recherche des lois de similitude des phénomènes instationnaires, $[6,7,8,9,10,11,12,13]$.

Le troisième domaine concerne la modélisation mathématique des phénomènes rencontrés. Elle ne consiste actuellement qu'en une série de modélisations partielles ouvrant la voie à une représentation globale de la réalité physique, $[14,8,15,12]$.

La présente communication prend place dans le deuxième domaine. Son objectif est de clarifier les relations entre stabilité hydraulique (au sens du couple moteur) et les fluctuations de pression, d'une part, et de séparer l'influence des diverses fréquences intervenant dans cette stabilité, d'autre part.

\section{Description rapide du vortex diphasique}

Le terme "torche" n'est utilisé ici qu'au sens visuel.

La torche est définie comme un vortex rendu visible par la phase vapeur engendrée par la basse pression. D'une machine à l'autre, son aspect varie beaucoup avec la charge et peu avec la chute :

- à très basse charge, se manifestent des tourbillons qui prennent naissance au plafond de la roue et se développent dans le canal entre aubes. $\mathrm{La}$ torche centrale, mal organisée, n'est que peu ou pas visible ;

- pour des débits plus élevés, mais toujours à charge partielle, apparait une torche de forme hélicoîdale toumant environ au quart de la vitesse de la roue et dans le même sens. La figure 1 en donne un aspect typique ;

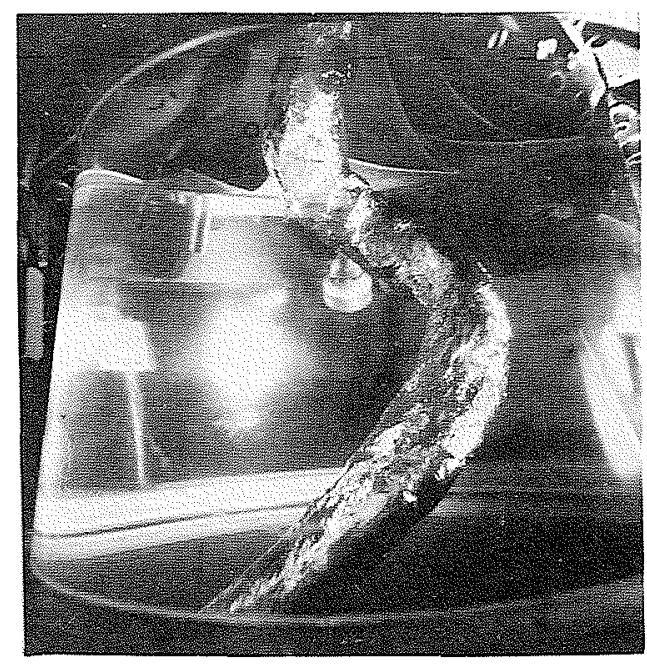

Figure 1 - Noyau en hélilce à charge partielle.

LA HOUILLE BLANCHE/ $\mathrm{N}^{\circ} 2 / 3-1982$ 
- l'aspect visuel de la torche dépend, dans une large mesure, de la vitesse spécifique et du tracé hydraulique. Ainsi, on connaît certains tracés pour lesquels le noyau présente une forme de révolution symétrique au lieu de la forme hélicoïdale attendue;

- l'expérience permet d'affirmer que la répartition moyenne dans le temps des vitesses méridiennes $\mathrm{Cm}$ et périphériques $C u$ à l'entrée de l'aspirateur est le facteur principal déterminant la forme du vortex et de son noyau, ainsi que son excentrement à charge partielle ;

- enfin, d'autres mouvements que le simple mouvement de rotation excentrée peuvent apparaître.

\section{Expérimentation et analyses concernant la sta- bilité sur modèle réduit}

\section{Définitions}

"Fluctuation" : ce terme désigne une variation de pression ou de vitesse périodique pour un observateur fixe, mais stationnaire pour un observateur se déplaçant selon la période en question. La figure 2 donne un exemple idéalisé de fluctuation dans le cône de l'aspirateur à charge partielle.

"Pulsation" : ce terme désigne une variation de pression ou de vitesse qui demeure périodique aussi bien pour un observateur fixe qu'un observateur mobile. La figure 2 montre ainsi le cas d'une pulsation avec vortex d'axe rectiligne provoquée, par exemple, par une surcharge de la roue ou une résonance avec un conduit.
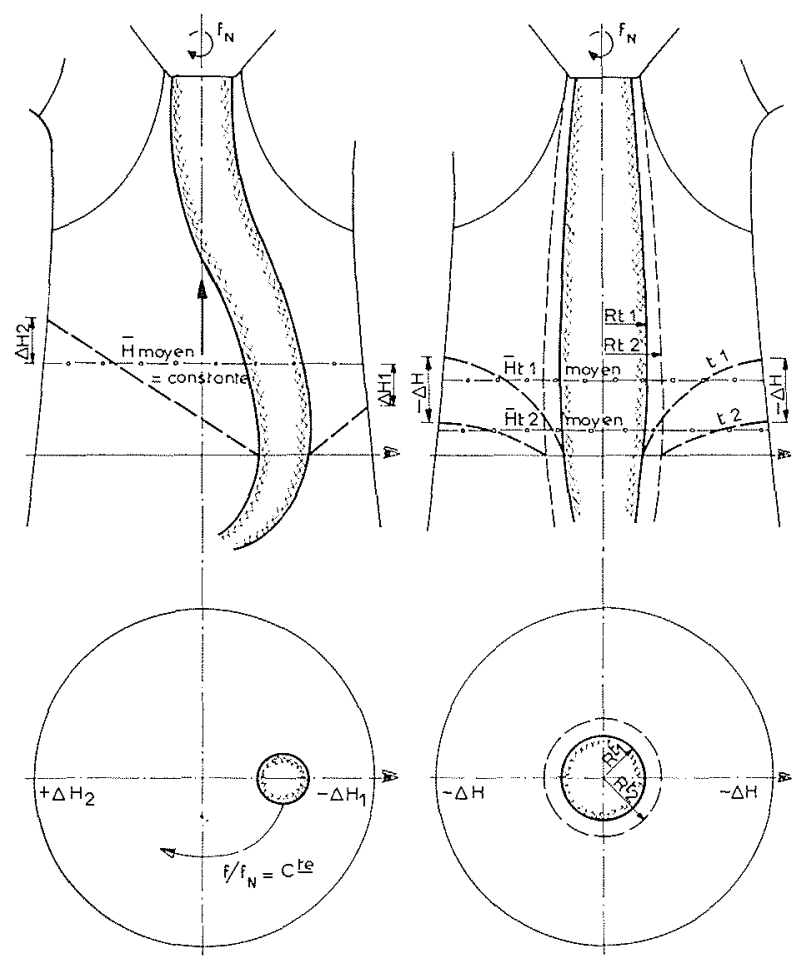

EXEMPLE DE FLUCTUATION

EXEMPLE DE PULSATION

Figure 2 - Définitions des phénomènes.
"Déformation" : il s'agit du cas limite de la fluctuation représentée figure 2 pour laquelle l'excentricité du mouvement du noyau du vortex devient nulle mais où subsistent des irrégularités provoquées par les singularités des aubes, du bec de bâche, etc.

"Stabilité" : une analyse expérimentale exige une définition pratique de la stabilité ; par exemple, selon des critères relatifs qui peuvent varier d'une turbine à l'autre :

- les fluctuations respectives de la puissance électrique, du couple hydraulique et de la pression, dans l'aspirateur ou la volute, ne doivent pas dépasser des limites fixées d'avance ;

- il ne doit pas y avoir d'implosions dans l'aspirateur, car elles provoqueraient des sauts de puissance ou des dégâts matériels inadmissibles.

La difficulté qu'on rencontre à fixer des valeurs limites de pulsation du couple moteur peut être partiellement écartée en complétant le critère sur l'amplitude par un critère sur le gradient du genre "on ne doit pas dépasser telle limite de fonctionnement au-delà de laquelle la pulsation du couple augmente rapidement". C'est ce qu'illustre la figure 3 .

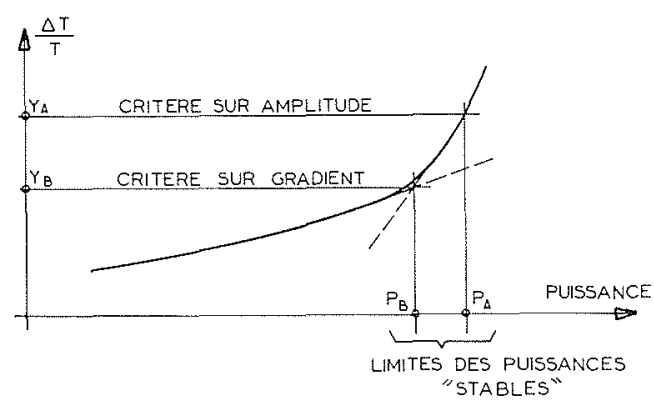

Figure 3 - Exemple de critères possibles de stabilité.

Pour mieux cerner les conditions de fonctionnement satisfaisant aux critères de stabilité du couple, la présente communication tente de répondre aux questions suivantes :

- comment évolue la pulsation de couple en fonction des autres mesures dynamiques?

- peuton se passer de la mesure du couple dynamique en considérant une ou plusieurs mesures simples de pressions instationnaires?

\section{Mesures sur modèle réduit}

Les grandeurs suivantes font l'objet de mesures continues :

Pression dynamique

- dans le cône de l'aspirateur, côtés amont et aval

$$
\Delta H_{1}, \text { resp. } \Delta H_{2}
$$

- dans la volute, partie basse de la section d'entrée

$$
\Delta H_{3}
$$

Couple dynamique sur l'arbre principal 
Poussée axiale dynamique (pression d'huile dans la chambre de butée)

\section{$\Delta F$}

Des observations visuelles et acoustiques complètent les mesures.

La turbine utilisée pour les essais est du type Francis, $n_{q} \sim 75$, diamètre de sortie du rotor : $0,400 \mathrm{~m}$.

Les essais sont exécutés en circuit fermé, partiellement dégazé (teneur en air $\sim 20 \%$ de la saturation), avec un seul niveau libre à l'aval de la turbine.

L'appareillage est constitué par des capteurs piézoélectriques pour la mesure des pressions et un torsiomètre à jauges de contraintes pour la mesure du couple. L'enregistrement est effectué simultanément sur bandes magnétique et photographique $(U V)$.

\section{Analyse des informations}

L'utilisation d'un analyseur analogique-digital pour traiter les signaux enregistrés sur la bande magnétique conduit à des analyses systématiques intéressantes. Dans le cadre de cette communication, seuls les résultats suivants ont été retenus :

- spectre amplitude/fréquence pour chacun des signaux enregistrés, c'est-à-dire la racine carrée de l'autospectre ;

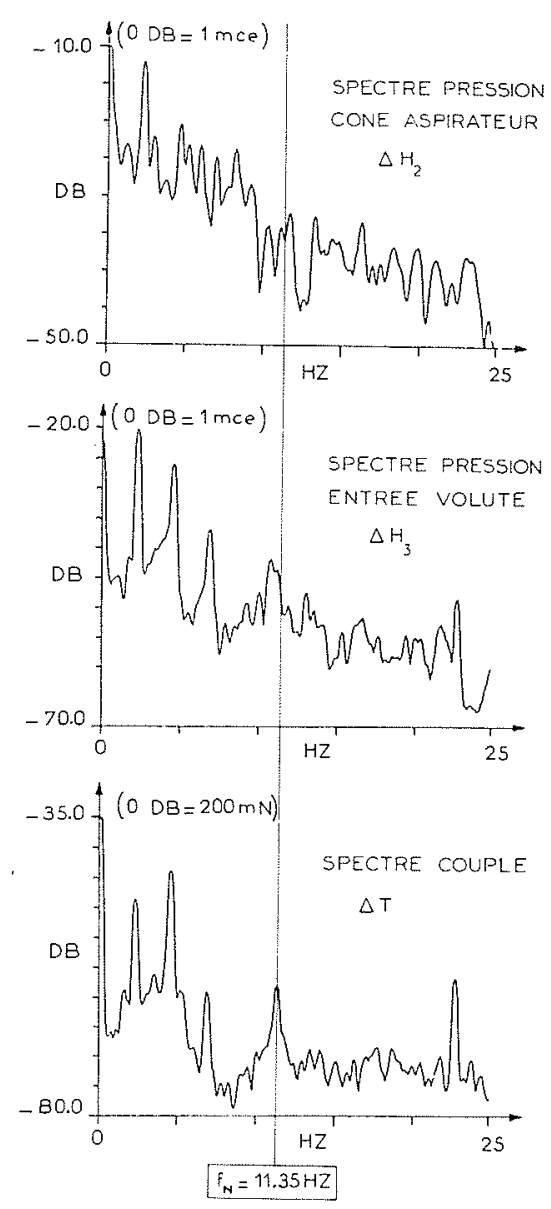

Figure 4 - Fréquences comparées des signaux

$$
\begin{aligned}
Q / Q_{n} & =0,715 & H_{\text {essai }} & =12 \mathrm{~m} \\
H / H_{n} & =1,04 & \sigma & =0,19
\end{aligned}
$$

- cohérence de chacun des signaux par rapport au signal $\Delta H_{1}$ du capteur amont dans le cône de l'aspirateur ;

- transfert d'amplitude et de phase de chaque signal par rapport à $\Delta H_{1}$.

La figure 4 donne un exemple des divers spectres fournis par l'analyseur pour un régime hydraulique de charge partielle avec noyau de cavitation atteignant le fond du coude de l'aspirateur.

La figure 5 présente des résultats de cohérence et de transfert pour le même régime hydraulique. Il apparaît clairement que la cohérence n'est pas uniformément assurée et qu'on ne peut donc interpréter les relations de transfert sans choisir les bandes de fréquences appropriées.

\section{Similitude dans l'expérimentation}

\section{Temps}

L'utilisation d'un temps relatif rapporté à la fréquence de rotation de la turbine est justifiée dans la mesure où les vitesses d'écoulement considérées restent proportionnelles à la vitesse de rotation. Cela revient à admettre

$$
t_{m} / t_{N_{m}}=t_{p} / t_{N_{p}}
$$

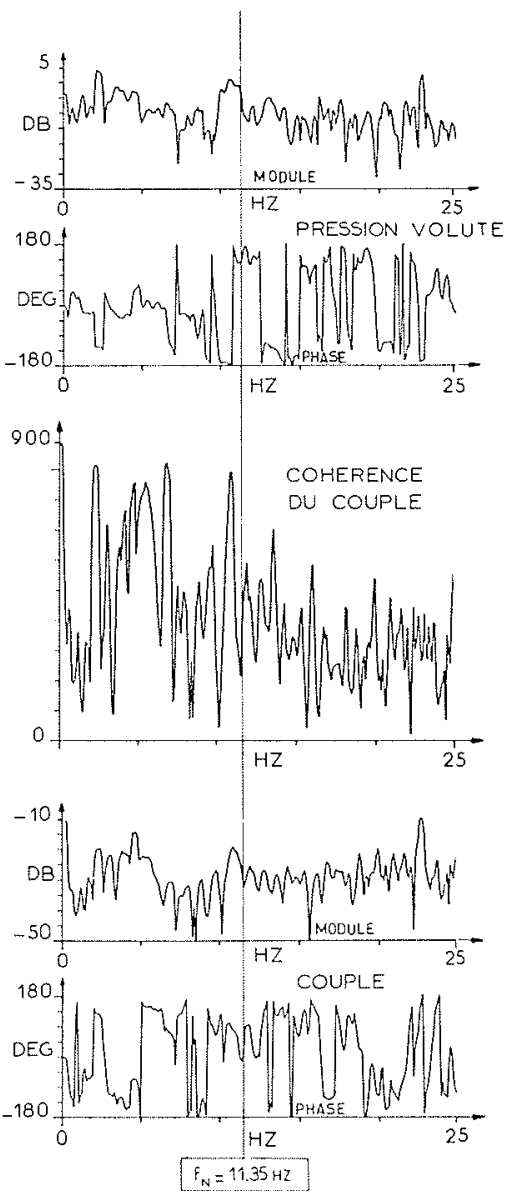

Figure 5 - Relations de transfert et de phase par rapport à la pression dans le cône aspirateur

$$
\begin{aligned}
Q / Q_{\wedge} & =0,715 & H_{\text {essai }} & =12 \mathrm{~m} \\
H / H_{n} & =1,04 & \sigma & =0,19
\end{aligned}
$$


bien que la célérité d'onde acoustique, indépendante de la vitesse de rotation, ne satisfasse pas à cette condition.

\section{Géométrie}

Pour une transposition correcte des résultats relatifs aux fluctuations, l'homologie géométrique des formes et de l'écoulement doit être respectée, plus particulièrement pour les angles à la sortie de la roue, la forme de l'aspirateur et les conditions à l'entrée et à la sortie du modèle réduit.

Ainsi

et, par conséquent

$$
L_{m} / D_{m}=L_{p} / D_{p}
$$

$$
\beta_{m}=\beta_{p}
$$

\section{Régime hors cavitation}

La similitude hydrodynamique du point de fonctionnement est assurée par les critères classiques qui peuvent s'écrire

$$
\phi=c^{\text {ste }}, \psi=c^{\text {ste }}
$$

ou

$$
n_{11}=\mathrm{c}^{\text {ste }}, Q_{11}=\mathrm{c}^{\mathrm{ste}}
$$

\section{Régime en cavitation}

La forme et les dimensions de la torche dépendent non seulement du régime hydrodynamique mais également du niveau absolu de pression qui règne dans le diffuseur. Par conséquent, lorsqu'on s'intéresse à des figures de cavitation qui ont dépassé leur "début théorique", la reproduction des phénomènes n'est théoriquement obtenue qu'en maintenant constant le sigma et en respectant la similitude de Froude $H_{m} / D_{m}=H_{p} / D_{\rho}$, simultanément

Cette dernière condition entraînant des chutes d'essais relativement basses, il est néanmoins possible d'adopter des chutes plus grandes, à condition de choisir un sigma $(\sigma)$ de référence approprié.

Enfin, la teneur en air de l'eau devrait être considérée puisqu'elle conditionne la phase gazeuse. Malheureusement, le respect d'une condition de similitude est difficile du fait que cette tencur cn air cst mal connue ou fortement variable pour le prototype.

\section{Fluctuations de pression}

La transposition des amplitudes et des fréquences correspondantes de fluctuations de pression découle des critères de similitude précédents :

$$
\begin{gathered}
\Delta H_{m} / H_{m}=\Delta H_{p} / H_{p} ; \\
f_{m} / f_{N_{m}}=f_{p} / f_{N_{p}} .
\end{gathered}
$$

\section{Couple}

Comme il n'est pas possible de réaliser un modèle réduit de l'ensemble du groupe hydroélectrique, aux bornes duquel on mesurerait la puissance électrique, on se limite à mesurer le couple dynamique sur l'arbre de la turbine. La transposition des fluctuations de couple ainsi mesurées est licite, pour autant que les fréquences propres de la ligne d'arbres soient largement supérieures aux fréquences mesurées, aussi bien sur modèle que sur prototype. Par bandes de fréquence, on a $\Delta T_{m} / T_{m}=\Delta T_{p} / T_{p}$.

Comme l'écart sur prototype, entre fréquences d'excitation hydraulique du couple et fréquences propres, est en général plus faible que sur modèle, on ne peut exclure, à priori, une certaine amplification des fluctuations relatives de couple, telles que $\Delta T_{m} / T_{m} \approx \Delta T_{p} / T_{p}$

Si l'on tient compte, en outre, du fait que les fluctuations de vitesse de rotation $\Delta N$, accompagnant les fluctuations de couple $\Delta T$, n'ont très probablement pas la même importance relative sur modèle et sur prototype, il vaut mieux adopter des critères de stabilité relatifs à la dérivée $\Delta T / T$ plutôt que des critères de stabilité basés sur la valeur absolue de $\Delta T / T$ (cf. Fig. 3).

\section{Circuit}

Des mesures effectuées sur le circuit en l'absence de turbine ont confirmé l'existence de fréquences "parasites". Elles ont pu être "éliminées" grâce à l'analyse analogique digitale.

\section{Types de similitude réalisés pour les essais}

Quand la cavitation n'en est qu'au stade de début, la seule similitude, entre modèle et prototype, permettant de conserver simultanément les gradients de pression et les pressions minimums est réalisée pour $\sigma=\mathrm{c}^{\text {ste }}$ et $H / D=\mathrm{c}^{\text {ste }}$.

Pour les conditions d'implantation habituelles, la cavitation est développée et la conservation des constantes $\sigma$ et $H / D$ ne garantit plus la conservation de l'intensité de cavitation.

Pour déterminer dans quelle mesure il en résulte des écarts sur la transposition des amplitudes, voire des fréquences, deux similitudes ont été testées sur modèle réduit en faisant varier la chute d'essai :

- la similitude $A$ avec $\sigma=c^{\text {ste }}$ pour trois chutes d'essai. Les mesures sont effectuées pour trois valcurs de $\sigma$ correspondant à trois développements caractéristiques de la torche sous la chute médiane de $12 \mathrm{~m}$ (longueur torche : 0 , mi-coude, fond du coude aspirateur :

\begin{tabular}{c|c|c}
$\sigma=0.30$ (Fig. 6) & 0.34 & 0,45 \\
$H=6 \mathrm{~m}, 12 \mathrm{~m}, 18 \mathrm{~m}$ & $6 \mathrm{~m}, 12 \mathrm{~m}, 18 \mathrm{~m}$ & $6 \mathrm{~m}, 12 \mathrm{~m}, 18 \mathrm{~m}$
\end{tabular}

- la similitude $B$ avec une longueur et un diamètre de torche visuelle constants pour trois chutes d'essais, donc sans condition sur sigma :

$$
\begin{aligned}
& \text { Longueur torche/profondeur coude aspirateur } \\
& 100 \% \text { (Fig. 7) } \mid \text { 50\% } \\
& \text { Hauteur de chute nette : } \mathrm{H} \\
& 6 \mathrm{~m}, 12 \mathrm{~m}, 18 \mathrm{~m}|6 \mathrm{~m}, 12 \mathrm{~m}, 18 \mathrm{~m}| 6 \mathrm{~m}, 12 \mathrm{~m}, 18 \mathrm{~m}
\end{aligned}
$$



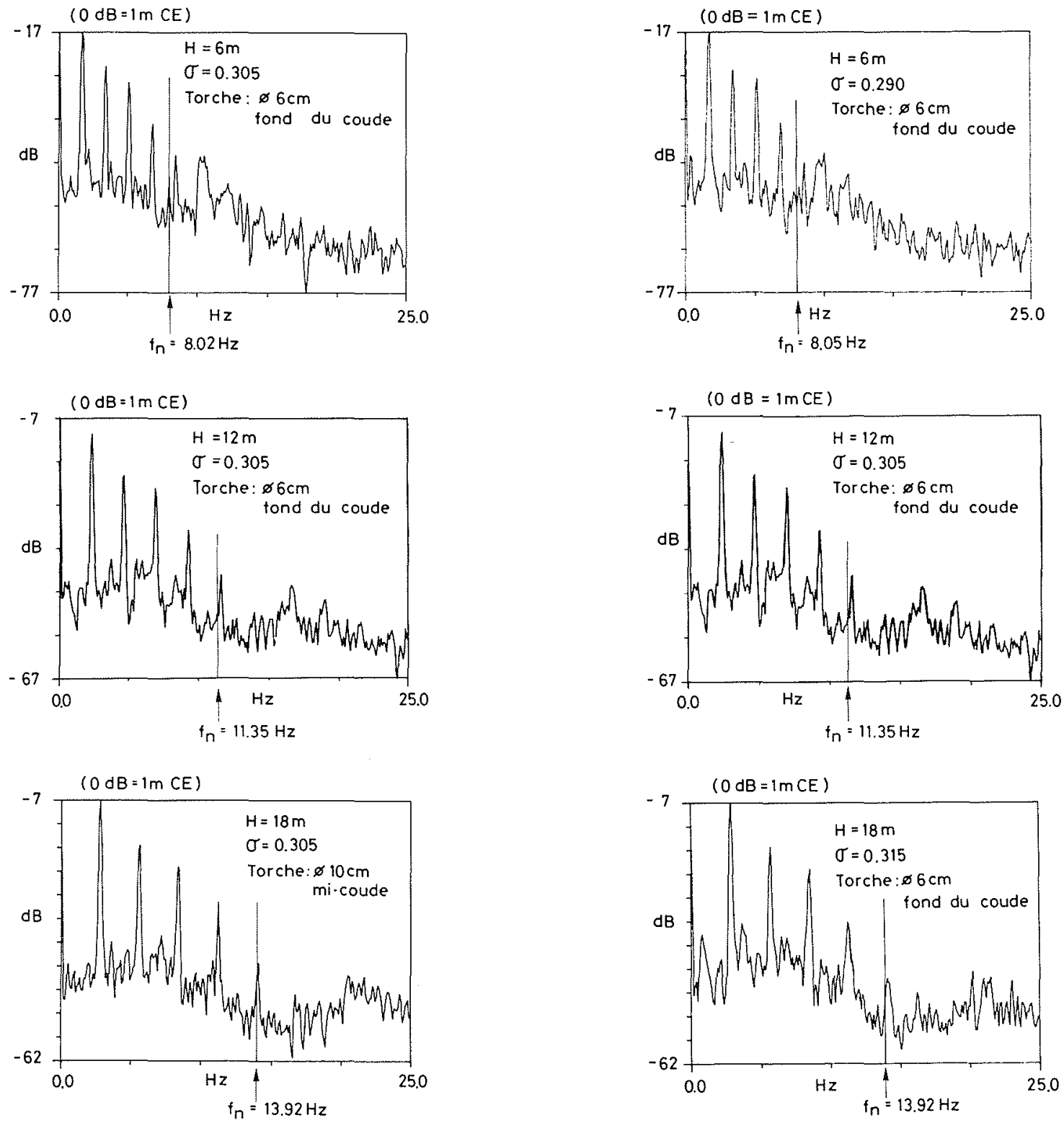

Figure 6 - Fréquences comparées en fonction de $H$ (similitude $A$, pression $\Delta H_{2}$ ).

Figure 7 - Fréquences comparées en fonction de $H$ (similitude $B$, pression $\Delta H_{2}$ ).

\section{Conclusions et synthèse}

Les conclusions portent sur :

- l'influence de la cavitation à chute constante ;

- l'influence de la chute avec les deux similitudes $A$ et $B$.

\section{Evolution des paramètres en fonction du degré de ca- vitation}

Les figures 8 et 9 présentent l'évolution des amplitudes et des phases de quatre signaux, dans leurs principales bandes de fréquences, pour un régime typique de charge partielle avec une chute d'essai constante égale à $12 \mathrm{~m}$.

Les fréquences rencontrées varient peu avec $\sigma$ et s'établissent comme suit :

\section{Fréquence 1 Fréquence 2 Fréquence 3

$\begin{array}{llll}f / f_{N} & 0.20 & 0.41 & 0.61\end{array}$

Ces différentes fréquences augmentent pour des régimes de cavitation très développés. Ainsi, pour $\sigma=0.0638$, on a $f / f_{N}=0.27,0.42$ et 0.68 et une nouvelle fréquence $f / \hat{f}_{N}=0.54$ devient perceptible.

Les amplitudes correspondantes varient sensiblement avec $\sigma$, comme en témoignent les observations suivantes :

- pour les $\sigma$ élevés, l'atténuation entre $\Delta H_{2}$ (cône) et $\Delta H_{3}$ (volute), très forte sur la fréquence 1 , subit une réduction sensible sur les fréquences 2 et 3 ;

- pour le $\sigma$ le plus bas, l'amplification très marquée sur $\Delta H_{2}$ à toutes fréquences devient au contraire une atténuation sur $\Delta H_{3}$ dans la volute ;

- l'évolution de $\Delta T$ est beaucoup plus voisine de 


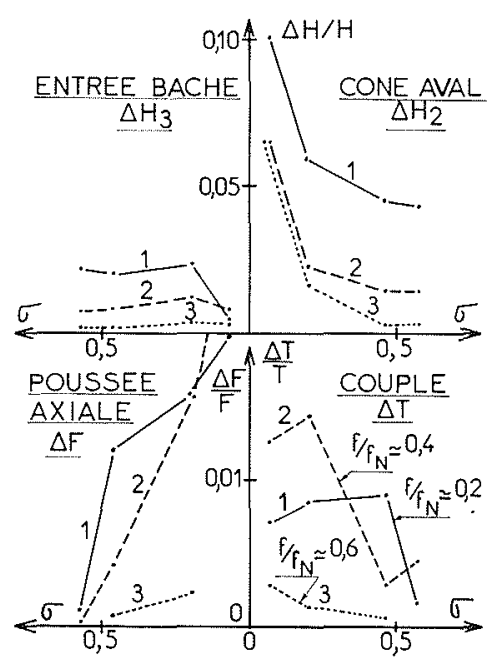

Figure 8 - Influence de la cavitation sur les amplitudes $Q / Q_{\wedge}=0,715 \quad H / H_{\wedge}=1.04$

celle de $\Delta H_{3}$ (volute) que de $\Delta H_{2}$ (aspirateur). Pour les $\sigma$ les plus bas, voisins des $\sigma$ d'implantation, le maximum de $\Delta T$ se manifeste sur la fréquence 2 ; - $\Delta F$ présente la plus forte variation en fonction du $\sigma$ avec un fort gradient d'amplitude pour la fréquence 2:

Les phases (par rapport au signal $\Delta H_{1}$ ) présentent des évolutions plus ou moins simples.

Ainsi, $\Delta H_{2}$ se tient globalement en opposition de phase avec $\Delta H_{1}$ sur la fréquence 1 et en parfait accord de phase avec $\Delta H_{1}$ sur la fréquence 2 .

Les phases du couple $\Delta T$ et de la pression volute $\Delta H_{3}$ présentent la même allure au signe près.

En revanche, d'autres évolutions de phases demandent plus d'analyse comme celles de $\Delta H_{3}$ comparées à celles de $\Delta H_{2}$.

Trois conclusions simples peuvent être tirées de ces analyses :

- la fréquence 1 correspond bien à un mouvement de giration excentré du noyau du vortex et ce mouvement entraîne plus une fluctuation locale de débit qu'une pulsation globale ;

- la fréquence 2 correspond au passage complexe du noyau du vortex dans le coude de l'aspirateur et ce mouvement entraîne une pulsation globale du débit. C'est ce mouvement qui provoque la majeure partie de la fluctuation du couple aux $\sigma$ les plus bas, voisins des $\sigma$ d'implantation;

- la forte augmentation des amplitudes de $\Delta H_{2}$ à très bas $\sigma$ n'est pas accompagnée d'une augmentation de la pulsation de débit. Il s'agit probablement d'une augmentation des fluctuations locales du débit qui s'organisent entre phase vapeur et phase liquide.

\section{Influence de la chute}

\section{Similitude $A$}

La similitude $A$ assure la constante des valeurs de $\sigma$ pour les trois chutes d'essais. Les $\sigma$ de référence sont ceux qui, sous $12 \mathrm{~m}$, correspondent à des torches

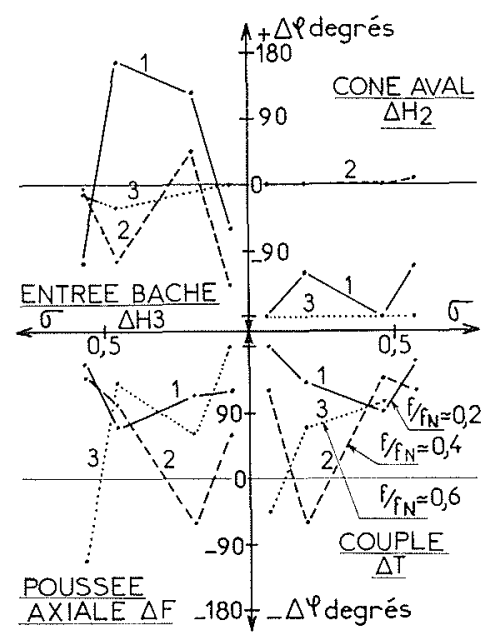

Figure 9 - Influence de la cavitation sur les phases $Q / Q_{n}=0,715 \quad H / H_{n}=1.04$

allant jusqu'au fond du coude, à mi-coude et à la torche apparaissant.

Les figures $10 a$ et $10 b$ résument les principales am. plitudes pour les deux premières fréquences caractéristiques des spectres. L'influence de la chute est faible pour $\sigma=0.30$ et 0.45 et pour $f^{\prime} / f_{N} \sim 0.2$. En revanche, le $\sigma$ intermédiaire de 0.34 conduit, sous $18 \mathrm{~m}$, à une quasi-disparition des amplitudes.

Dans la gamme $f^{\prime} / f_{N}^{\prime} \sim 0.4$, on retrouve l'absence d'influence significative de la chute pour les $\sigma$ extrêmes 0.3 et 0.45 . Avec $\sigma=0.34$ et $H=18 \mathrm{~m}$, apparait une baisse très nette des amplitudes $\Delta H_{1} / H$ et $\Delta H_{2} / H$ mais qui ne se répercute, ni sur $\Delta H_{3}$, ni sur $\Delta T$, ni sur $\Delta F$.

\section{Similitude $B$}

La similitude $B$ assure mieux la constance visuelle des dimensions du noyau gazeux lorsque la chute varie. La valeur de $\sigma$ est ajustée pour retrouver sous chaque chute les trois figures de torche décrites plus haut.

Les figures $11 a$ et $11 b$ résument les résultats d'amplitudes pour $f / f_{N} \sim 0.2$ et $f / f_{N} \sim 0.4$.

Pour $f / f_{N} \sim 0.2$, l'influence de la chute est peu marquée, sauf pour la torche à mi-coude sous $12 \mathrm{~m}$ qui entraîne un maximum très net sur $\Delta H_{1}$ et $\Delta H_{2}$ mais fortement atténué sur $\Delta H_{3}$ et $\Delta T$.

Pour $f / f_{N} \sim 0.4$, on retrouve le maximum sous $12 \mathrm{~m}$ pour la torche à mi-coude, mais ce maximum disparaît sur $\Delta T$. A noter également une certaine amplification pour $H=6 \mathrm{~m}$ et pour la torche la plus longue, cette amplification étant bien marquée sur $\Delta H_{3}, \Delta T$ et $\Delta F$

\section{Synthèse et perspective}

L'influence de la cavitation à chute constante est particulièrement nette sur trois points :

- la stabilité rapportée au couple diminue aux basses valeurs de $\sigma$ mais sur la deuxième fréquence du spectre et non sur la première fréquence correspondant au 

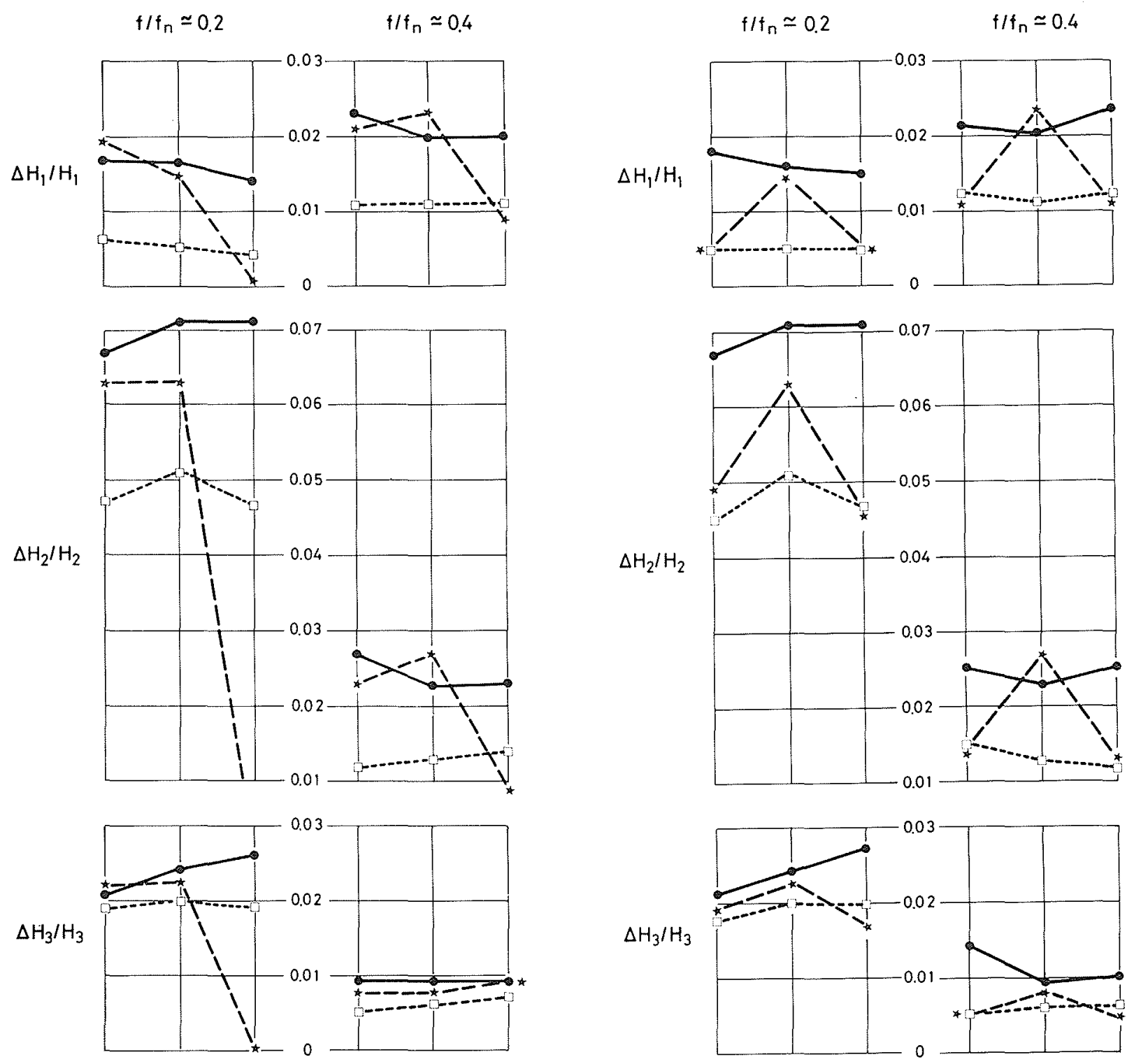

$\Delta T / T$

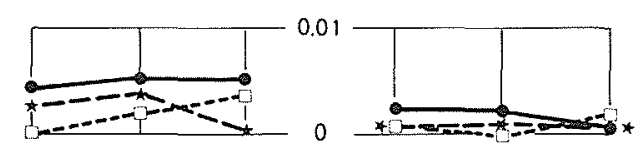

$\Delta T / T$
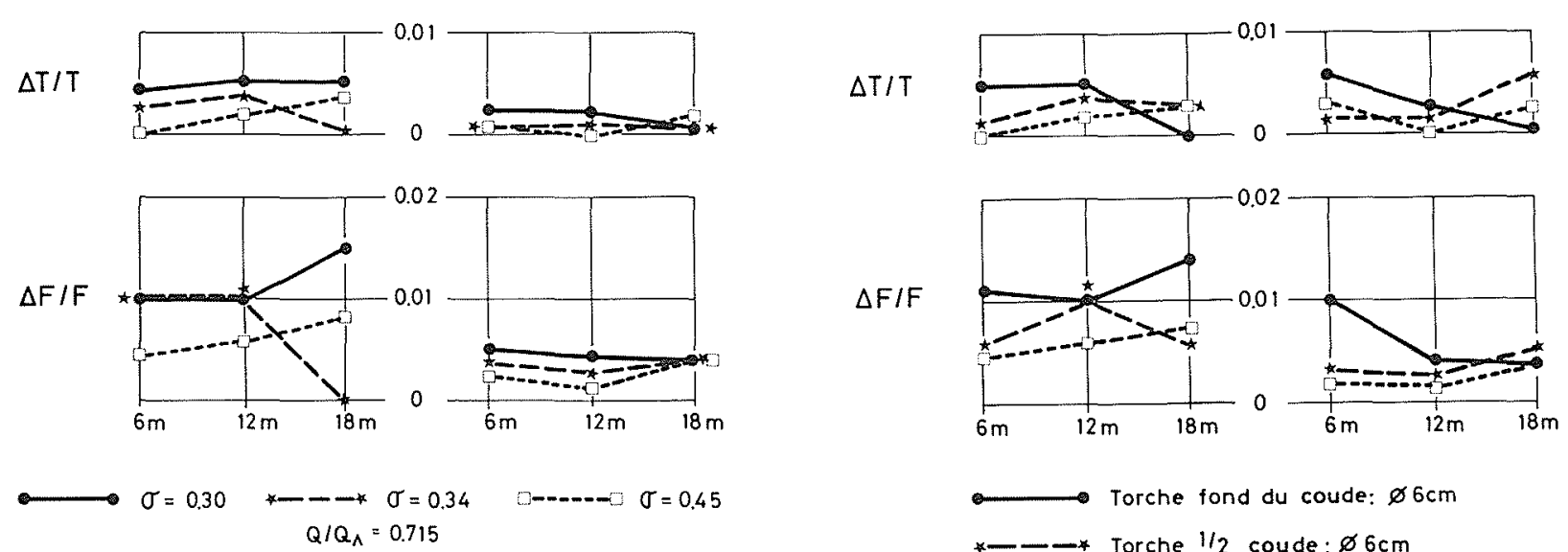

Figure 10 - Amplitudes comparées en fonction de $H$ (similitude $A$ ).

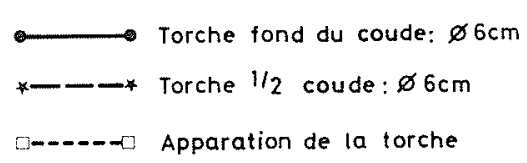

$Q / Q_{\wedge}=0.715$

Figure 11 - Amplitudes comparées en fonction de $H$ (similitude $B$ ). 
mouvement de giration excentrée du noyau de vapeur. - la baisse de $\sigma$ n'entraîne pas sur le couple les mêmes gradients d'amplitudes que sur les pressions, ni en valeur, ni en sens. L'évolution du couple dynamique se rapproche plus de l'évolution de la pression dynamique dans la volute que de celle de la pression dans l'aspirateur.

- les deux premières fréquences des spectres varient peu avec $\sigma$ et correspondent d'une part, au mouvement de rotation excentrée dans le cône, et d'autre part, au mouvement complexe du noyau à la sortie du coude. Le premier mouvement se caractérise par le déphasage de $180^{\circ}$ entre deux mesures de pressions diamétralement opposées dans le cône, le deuxième mouvement entraîne une mise en phase de ces mesures.

Les essais de variation de la chute posent autant de questions qu'ils en résolvent... Les trois connaissances acquises ici peuvent s'énoncer ainsi :

- pour des valeurs de $\sigma$ relativement élevées, l'influence de la chute à $\sigma$ constant est faible et indéterminée. En particulier la chute la plus basse n'entraîne pas une minimisation des fluctuations ;

- pour des valeurs de $\sigma$ relativement élevées, les similitudes $A$ et $B$ ne donnent pas de résultats fort différents, mais il faut souligner que les noyaux de vapeur considérés ici restent de dimensions modestes; - le noyau de vapeur "à mi-coude" représente une figure de cavitation particulièrement sensible puisqu'il entraîne une singularité dans les résultats pour la chute $18 \mathrm{~m}$ avec la similitude $A$ et pour la chute $12 \mathrm{~m}$ avec la similitude $B$.

En perspective, il apparaît assez clairement que l'influence de la chute doit être testée à des stades de cavitation plus développés lorsque le noyau de vapeur atteint et dépasse la sortie du coude. Si l'on veut continuer à comparer les similitudes $A$ et $B$, il conviendra de mettre en place des instruments de visualisation plus performants. D'autre part, la cinétique du noyau à la sortie du coude sera précisée par des moyens optiques ainsi que par des mesures denses de pressions en paroi. Enfin, on devra vérifier les incertitudes extrêmes que peut entraîner la variation de la teneur en air.

\section{Notations}

\begin{tabular}{lll}
$\begin{array}{ll}\text { Symbole } \\
H\end{array}$ & Unité & \multicolumn{1}{c}{ Grandeur } \\
$H_{x}$ & $\mathrm{~m}$ & $\begin{array}{l}\text { Hauteur de chute nette } \\
\text { Hauteur de colonne d'eau représen- } \\
\text { tative de la pression au point } x(x= \\
1,2,3)\end{array}$ \\
$\Delta H_{x}$ & $\mathrm{~m} \quad \begin{array}{l}\text { Hauteur de colonne d'eau représen- } \\
\text { tative de la variation dynamique de } \\
\text { la pression au point } x \quad(x=1,2,3), \\
\text { valeur crête à crête }\end{array}$ \\
$\begin{array}{lll}\text { Variation dynamique relative de la } \\
\text { pression, valeur crête à crête }\end{array}$ \\
$N$
\end{tabular}

$f_{N} \quad \mathrm{~Hz} \quad$ Fréquence correspondant à la vitesse

de rotation $N: f_{N}=N / 60$

$f / f_{N} \quad-\quad$ Fréquence relative

$t$ s Temps

$T \quad \mathrm{mN} \quad$ Couple

$\Delta T \quad \mathrm{mN}$ Variation dynamique du couple valeur crête à crête

$\Delta T / T$ - Variation dynamique relative du couple valeur crête à crête.

\section{$P \quad \mathrm{~W} ; \mathrm{kW}$ Puissance}

$F \quad$ N Poussée axiale

$F_{r} \quad \mathrm{~N} \quad$ Poussée axiale de référence: $F_{r}=$ 424.H

$\Delta F \quad \mathrm{~N} \quad$ Variation dynamique de la poussée axiale, valeur crête à crête

$\Delta F / F_{r} \quad-\quad$ Variation dynamique relative de la poussée axiale, valeur crête à crête

L m Longueur

D m Diamètre

$\sigma \quad$ - Chiffre de cavitation de Thoma

a/a. - Ouverture relative du distributeur.

Indices

^ Régime de rendement optimum

$m \quad$ Modèle

$p \quad$ Prototype

\section{Régimes instationnaires - bibliographie}

[1] CAMPMAS P. - Stabilité du régime de fonctionnement des turbines Francis. AIRH, Symposium Nice, 1960. Communication B4.

12] CAMPMAS P. et GIRAUD H. - Analyse du fonctionnement d'une turbine Francis. Comparaison des investigations sur modèle réduit et sur turbine industrielle. $A I R H$, Symposium Nice, 1960. Communication B6.

[3] DZIALLAS R. - Francis Turbinen bei Teil - und Uberlast. VDI-Berichte, Nr. 75, 1964, p. 53-64.

[4] GREIN H. et JAQUET M. - Measurements of pressure fluctuations in storage pumps and pump turbines. AIRH Symposium Rome, 1972. Communication H1, p. 1-12.

[5] SCHLEMMER G., GERICH R., RAABE J. - Measurement of unsteady flow field in Francis turbines of high specific speed. AIRH, Symposium Rome, 1972. Communication H3.

[6] DERIAZ P. - A contribution to the understanding of flow in draft tube of Francis turbines. $A I R H$, Symposium Nice, 1960. Communication B1.

[7] GREIN H. - Partial pressure and gaz content inside a cavitating vortex core and influence on pressure fluctuations in a draft tube of a Francis type pump-turbine. Proceedings of the conference on cavitation, C182, 1974. Tiré-à-part Escher Wyss, Zurich.

[8] CASSIDY J.J. and FALVEY H.T. - Observations of unsteady flow arising after vortex breakdown. J. Fluid mech, 1970, vol. 41, part 4, p. 727-736.

[9] GREIN H. - Vibration phenomena in Francis Turbines : their causes and prevention. AIRH, Symposium Tokyo, 1980, p. 527-539.

[10] FISCHER R.K., PALDE U. and ULITH P. - Comparison of draft tube surging of homologous scale models and prototype Francis turbines. AIRH, Symposium Tokyo, 1980, p. 541-556. 
[11] ULITH P., JAEGER E.-U. and STRSCHELETZKY M. Contribution to clarifying the inception of nonstationary flow phenomena in the draft tube of high specific speed Francis turbines operating at part load. AIRH, Symposium Vienne, 1974. Communication III4, p. 1-18.

[12] PALDE U.J. - Influence of draft tube shape on surging characteristics of reaction turbines. REC-ERC. 72-24, U.S. Bureau of Reclamation, Denver, 1972.

[13] HOSOI Y. - Contribution to model tests of draft tube surges of Francis turbines. AIRH, Symposium Tokyo, 1980, p. $141-150$.

[14] DÖRFLER P. - Modèle mathématique des oscillations excitées à charge partielle par la torche de cavitation dans les turbines Francis. Bulletin Escher Wyss, 1980, $\mathrm{n}^{\circ} 1 / 2$, p. 101-106.

[15] BRENNEN C. and ACOSTA A.J. - Theoretical, Quasistatic analysis of cavitation compliance in turbopumps. Journal of Spacecraft, 1973, vol. 10, no. 3, mars, p. $175-180$.
[16] HASHIMOTO H. - Swirling flow accompanied by cavity in circular tube. Rep. Ints. High sp. mech. Japan, vol. 19, 1967/1968, no. 241-257.

[17] NISHI M., KUBOTA T., MATSUNAGA S. and SENOO Y. - Study of swirl flow and surge in an elbow draft tube. AIRH, Symposium Tokyo, 1980, p. 557-568.

[18] CASACCI S., WEGNER M. GUITON P. - Analyse des vibrations et des instabilités de fonctionnement dans les turbines-pompes. La Houille Blanche, 1971, $\mathrm{n}^{\circ}$ 6, p. 519 530 .

[19] ZIELKE W. - Periodische Schwingungen und Resonanzerscheinungen in Druckrohrleitungen. Elektronische Berechnung von Roh- und Gerinneströmungen, 1974. p. 233-275.

[20] LECHER W. and BAUMANN K. - Francis turbines at part-load with high back pressure. AIRH, Symposium Lausanne, 1968. Communication B4, p. 1-12.

[21] FANELLI M. - Further considerations of the dynamic behaviour of hydraulic turbo-machinery. Water Power, 1972, juin.

\section{Discussion}

M. ROTH a observé lors d'une étude récente, en collaboration avec un constructeur, des figures de vortex un peu différentes de celles présentées par M. WEGNER, la torche commençant à l'intérieur de la roue. Ces résultats ont été corroborés par un calcul tridimensionnel par éléments finis.

M. ROSSI projette une diapositive qui compare quelques valeurs de pulsation mesurées par les auteurs avec d'autres mesurées dans les laboratoires Hydroart en des conditions presque équivalentes. Il remarque en particulier, comme dans ce cas, que la pulsation à la fréquence de la fondamentale est nettement prédominante sur les autres, de sorte que celle-ci seulement peut en pratique être assumée pour représenter le phénomène.

M. WEGNER fait observer que les divergences ne sont pas aussi importantes qu'on pourrait le croire, qu'il faudrait étendre un peu la plage de variation de $\sigma$ et étudier précisément les conditions expérimentales pour pouvoir faire une comparaison fructueuse, et que le premier harmonique des fluctuations de pression est effectivement renforcé dans les fluctuations de couple.

M. ZANETTI fait quelques considérations sur la possibilité d'interpréter le phénomène global d'instabilité aux charges partielles dans une turbine Francis en recourant à deux hypothèses simples : la première, que l'effet de la torche puisse être schématisé en une perte de charge périodique ayant lieu dans le coude de décharge, la seconde, que l'inertie de la conduite intervienne dans le phénomène. On montre un diagramme d'où l'on peut déduire les corrélations en amplitude et phase de quelques grandeurs intéressées au phénomène : pulsation de chute, de débit, de couple, etc.

M. CASACCI estime qu'il faut étudier séparément chaque partie de l'installation et en particulier approfondir notre connaissance de la fonction de transfert de la roue. De plus, il faut distinguer les pulsations globales et les pulsations locales car elles seront transmises tout à fait différemment par la roue.

\section{Intervention de M. GRAESER}

En régime permanent, la mesure du couple, à vitesse et ouverture du distributeur constantes, ne pose aucun problème et la transposition au prototype des valeurs moyennes mesu- rées sur modèle est régie par des lois de similitudes bien connues.

Quand il s'agit d'évaluer l'instabilité de la puissance qui se manifeste en régime permanent pour certains domaines de fonctionnement, seule la fluctuation du couple est mesurée. La fluctuation de vitesse qui lui est liée n'est pas connue. Il n'est donc pas encore possible de déceler dans quelle mesure la réaction dynamique du modèle aux sollicitations hydrauliques a une influence sur les fluctuations mesurées du couple.

A l'intérieur de la machine, les ondes de pression se propagent avec une célérité qui n'obéit pas à la loi de similitude des vitesses admises communément pour la transposition des résultats de mesure du modèle au prototype. L'erreur qui en résulte devrait être évaluée.

Enfin, les masses tournantes des gros prototypes constituent des systèmes élastiques (vibrations à la torsion, voir Fig. 12) susceptibles d'entrer en résonance à certaines fréquences de la fluctuation de couple. La prévision de tels phénomènes est indispensable au constructeur.

L'étude de ces problèmes, qui fera appel aussi bien à la mesure qu'au calcul, s'inscrit dans le développement de la recherche poursuivie par les auteurs de la communication.

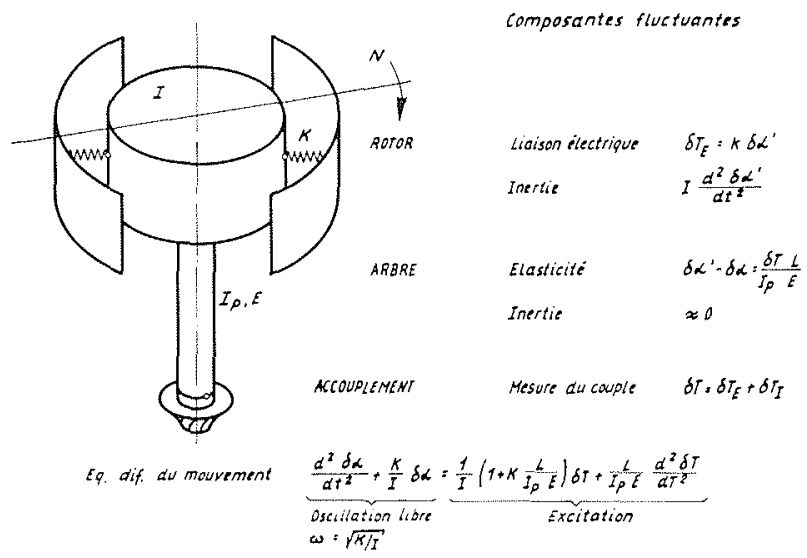

Figure 12 - Comportement des masses tournantes $\alpha, \alpha^{p}$ décalage angulaire par rapport à la référence du champ tournant. 


\section{Abstract \\ Experimental analysis of hydraulic stability of the partial load Francis turbine}

The paper begins by recalling briefly the general trends observed in the literature and the meaning of stability. Taking that the torque fluctuation on the main shaft is the most synthetic index of turbine stability into account, the paper deals, on the one hand, with the relationship between the pressure fluctuation in the draft tube cone and spiral casing, and on the other, the torque fluctuation. Fluctuations are measured on a Francis turbine model.

After recording the a.m. signals on tape, automatic analysis is carried out by use of a digital-analogic analyser which delivers the following information:

-.. frequency spectrum of each signal;

- consistency of each signal in relation to the other;

- amplitude transfer between two signals;

- phase transfer between two signals.

Two main parameters govern these model tests:

- the first one is the degree of cavitation with a constant operating head;

- the second one is the operating head keeping as a constant either the cavitation number or the dimension of the cavitating dead water core.

Main results and conclusions are described below:
- When cavitating dead water core increases, pressure fluctuations in the draft tube cone are aplified for all frequencies. Pressure fluctuations in the spiral casing are largely reduced for the dominant frequency, but much less for the first harmonic. As a result, the highest torque fluctuations are found for the first harmonic and not for the dominant frequency. - It therefore appears that the dominant frequency is produced by a local flow phenomenon (eccentric rotation of cavitating dead water core) and that the first harmonic is produced by a complete flow pulse due to the complex movement of the core in the draft tube elbow. The analysis of phases confirms that statement.

- When the operating head changes, keeping as a constant either the cavitation value or the size of the vapor value, the relative values of measured fluctuations do not have any significant trend. It can be observed that the lowest operating head does not lead to the lowest relative amplitudes of fluctuations. Nevertheless, observed dimensions of cavitating dead water core are not sufficiently developed to be able to draw a firm conclusion as to the effect of the operating head. The authors are therefore preparing an extension of the measurements to visual observatons in the draft tube elvow and analysis. 